\title{
Alternaria alternata as the cause of decline and necrosis on olive tree cuttings in Greece
}

\author{
George T. Tziros ${ }^{1}$ (D) - Antonios Karpouzis ${ }^{1} \cdot$ Anastasia L. Lagopodi $^{1}$
}

Received: 30 October 2020 / Accepted: 22 February 2021 / Published online: 4 March 2021

(c) Australasian Plant Pathology Society Inc. 2021

\begin{abstract}
In 2018, decline and necrosis were observed on olive tree cuttings (Olea europaea) in a nursery in northern Greece. The pathogen was identified as Alternaria alternata on the basis of conidia morphology and sequence analysis of the internal transcribed region (ITS) and part of the elongation factor 1a (EF-1a). The endopolygalacturonase (endoPG) and the Alternaria major allergen (Altal) genes were also amplified. A pathogenicity test was performed and Koch's postulates were confirmed by re-isolation of the fungus $A$. alternata from artificially inoculated cuttings. This is the first report of $A$. alternata as the cause of decline and necrosis on olive tree cuttings in Greece.
\end{abstract}

Keywords Olea europaea $\cdot$ Alternaria alternata $\cdot$ Sequence analysis $\cdot$ Pathogenicity

Olive tree (Olea europaea) is the emblematic plant species of the Mediterranean Basin and its cultivation is well adapted to the environmental conditions prevailing in the specific area (Torres et al. 2017). According to the report of the International Olive Oil Council (IOC 2012) more than $70 \%$ of the globally cultivated olive trees are located in European Union's Mediterranean countries with Greece ranked as the third producer country in the world. It has an average annual production of 285,000 tons of olive oil, following Spain and Italy. Olive cultivation is one of the most significant agricultural activities in Greece and a major contributor to the country's economy.

The propagation of olive trees is a key factor to the success of new plantations or the renovation of the existing orchards. Olive plants are propagated from shoot cuttings in commercial orchards, a technique which ensures, in a short time, the production of uniform new plants of the same varietal origin.

In June 2018, several declining leafy cuttings of olive tree shoots (Olea europaea, cv. Kalamon) were observed in a commercial nursery in Nea Raidestos, in the Prefecture of Thessaloniki (Northern Greece, Central Macedonia).

George T. Tziros

gtziros@yahoo.gr

1 Faculty of Agriculture, Laboratory of Plant Pathology, Aristotle University of Thessaloniki, 54124 Thessaloniki, Greece
Cuttings cut at both edges, derived from healthy olive shoots, were maintained for rooting in plastic boxes under mist conditions. The disease was severe and widespread resulting in approximately $30-40 \%$ mortality of approximately 500 plants. The symptoms consisted of dark brown lesions visible on the surface of the cuttings' upper part, which in most case was made at an angle. Infected areas gradually enlarged in size (Fig. 1), became irregular in shape, and in some cases were sunken or surrounded the cutting top, while leaves remained symptomless. The infection expanded in the woody tissue, under the epidermis, in approx. $0.5-1 \mathrm{~mm}$ depth (Fig. 1). At a later stage of infection, complete necrosis of infected cuttings and subsequent shedding of leaves were observed.

The causal agent of the disease was successfully isolated on potato dextrose agar (PDA) from the diseased cuttings. Small pieces of tissue from the margins between healthy and diseased tissue, below the epidermis, were disinfested in $1 \%$ sodium hypochlorite for 1 min, plated on PDA and then incubated for 7 days, at $24^{\circ} \mathrm{C}$, with a $12 \mathrm{~h}$ photoperiod. For the identification of the pathogen single spore cultures, derived from the isolates, were sub-cultured on potato carrot agar (PCA) (Simmons 2007). These cultures gave rise to initially white colonies which turned grayish-black later due to abundant sporulation. Conidiophores were short, septate, branched or unbranched, and green to brown in colour. Conidia were obclavate, obpyriform or ellipsoidal with a short beak, showing 1 to 5 transverse and 0 to 3 longitudinal 
Fig. 1 Necrosis at the upper part of olive cuttings: a Initial stage of infection, $\mathbf{b}$ Later stage of infection
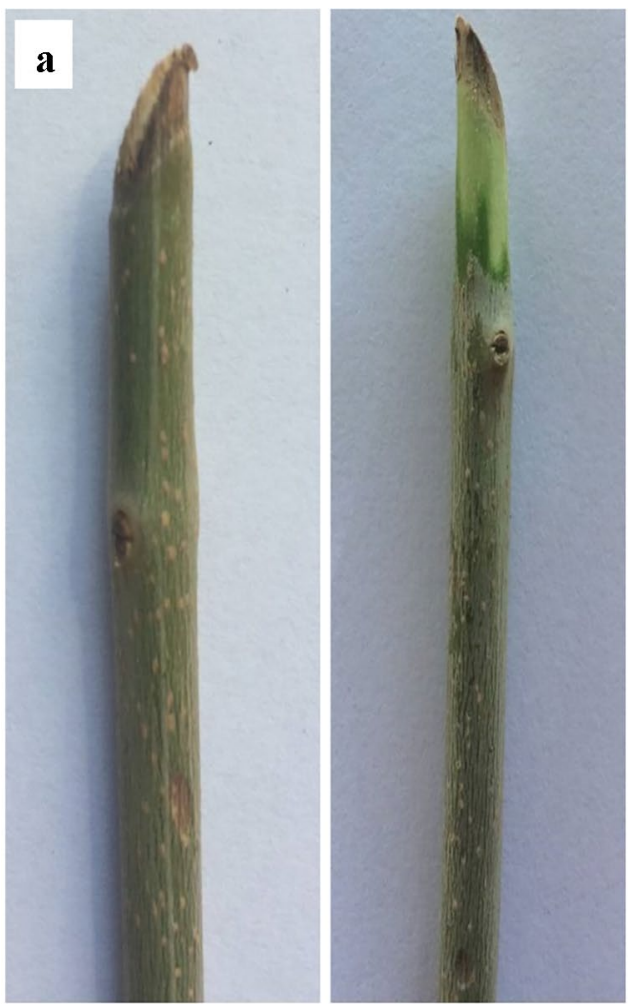

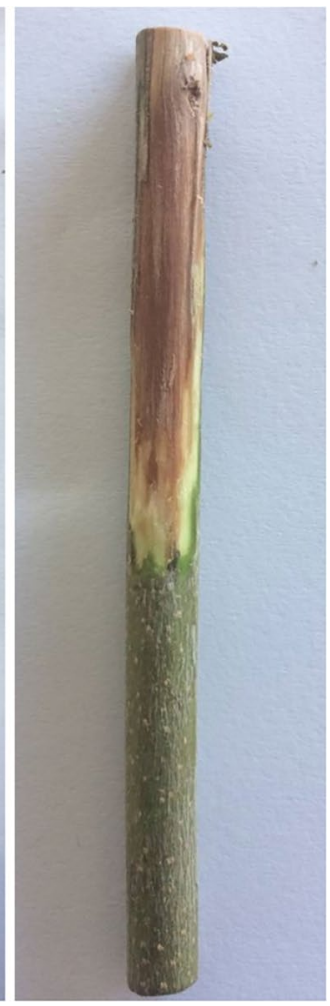

septa and measured 23.9-53.1 $\times 16.8-23.9 \mu \mathrm{m}$ (average 42.3-21.8 $\mu \mathrm{m}$ ). The morphology of hyphae and conidia and the dimensions of the spores concurred with those of Alternaria alternata (Simmons 2007). One representative isolate was deposited at the Benaki Phytopathological Institute Culture Collection (Athens, Greece) as BPIC 2709 (Table 1).

The ITS1-5.8S-ITS2 region of three single spore isolates was amplified with primers ITS1/ ITS4 (White et al. 1990), while partial amplification of elongation factor 1-alpha (EF1) was performed with EF1-728F/ EF1-986R primers (Carbone and Kohn 1999). The endopolygalacturonase (endoPG) and the Alternaria major allergen (Altal) genes of

Table 1 Isolates of Alternaria alternata used in this study and GenBank Accesion Numbers

\begin{tabular}{lllll}
\hline Isolate & \multicolumn{4}{l}{ GenBank Accession Numbers } \\
\cline { 2 - 5 } & ITS & EF-1a $^{\mathrm{a}}$ & endoPG $^{\mathrm{b}}$ & Alta1 $^{\mathrm{c}}$ \\
\hline AOC1 $^{\mathrm{d}}$ & MN245978 & MN477952 & MN524513 & MN512439 \\
AOC2 & MN245979 & MN477953 & MN524514 & MN512440 \\
AOC3 & MN245980 & MN477954 & MN524515 & MN512441 \\
\hline
\end{tabular}

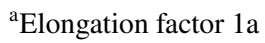

bendopolygalacturonase

${ }^{\mathrm{c}}$ Alternaria major allergen

${ }^{\mathrm{d}}$ Isolate deposited as BPIC 2709 the three isolates were additionally amplified using specific primers PG3/PG2b (Andrew et al. 2009) and Alt-for/Alt-rev (Woudenberg et al. 2015), respectively. All the amplicons were directly sequenced and deposited in GenBank under the Accession Numbers presented in Table 1. Maximum likelihood analysis based on the combined four genes in MEGA7 showed that sequences of these three strains were clustered with Alternaria alternata, supported by a $100 \%$ bootstrap value (Fig. 2). This phylogenetic analysis based on multigenes indicated that the studied isolates and other A. alternaria isolates formed one clade. The studied isolates clustered with ex-type strain A. alternaria CBS 916.96 and other known strains but not with other Alternaria species (Woudenberg et al. 2015). A BLAST search revealed 99-100\% identity with various A. alternata isolates (e.g. MN394880 for ITS, MK791316 for EF1, MH975219 for endoPG and MK722048 for Altal). Therefore, the pathogen was identified as A. alternata on the basis of the morphological characteristics and sequence analysis of one genomic region and three different genes.

For pathogenicity tests, the above three isolates obtained from diseased tissue, grown on PCA for 2 weeks, were used to artificially inoculate healthy cuttings of $O$. europaea. Because the infection was observed in connection with the upper cut edge of the cuttings, a wounding technique was used to artificially inoculate the plants (Dhingra and Sinclair 1995). PDA plugs, $4 \mathrm{~mm}$ in diameter, with actively growing mycelia were transferred on shallow (less than $0.5 \mathrm{~mm}$ ) wounds made by a 
Fig. 2 Phylogenetic tree generated in MEGA7 and inferred from the concatenated alignments of ITS, EF-1a, endoPG and Alta1 gene sequences from fungi of the genus Alternaria by the Maximum Likelihood method, with 1000 bootstrap values. Values of more than $50 \%$ are presented on the branch. The studied isolates are indicated with a black dot. Branch length is proportional to the numbers of nucleotide substitutions as indicated by the scale bar

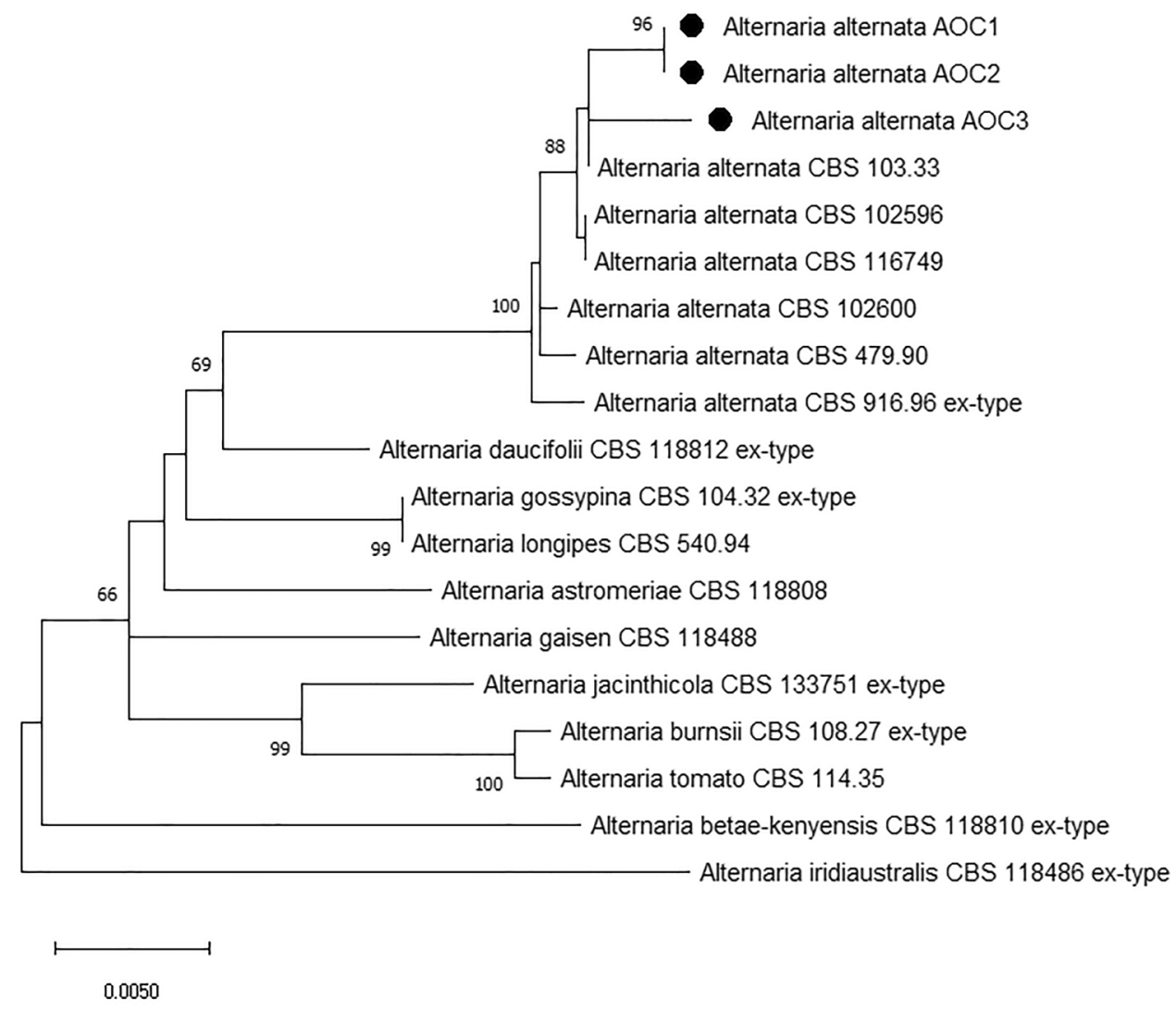

sterile scalpel on the epidermis of previously sterilized shoots. Inoculum was kept in place using parafilm. Each treatment consisted of ten cuttings and the pathogenicity test was repeated three times. Cuttings inoculated in the same way using PDA disks were kept as controls. Following inoculation, cuttings were sprayed with sterile water and incubated in a growth chamber at $20^{\circ} \mathrm{C}(60-70 \%$ relative humidity and $16 \mathrm{~h}$ photoperiod). Three weeks after inoculation visible lesions developed on all inoculated cuttings, while controls remained symptomless (Fig. 3). Infection development was evaluated by measuring
Fig. 3 Necrotic lesions on artificially inoculated Olea europaea cuttings: a Control, b Cuttings inoculated with Alternaria alternata

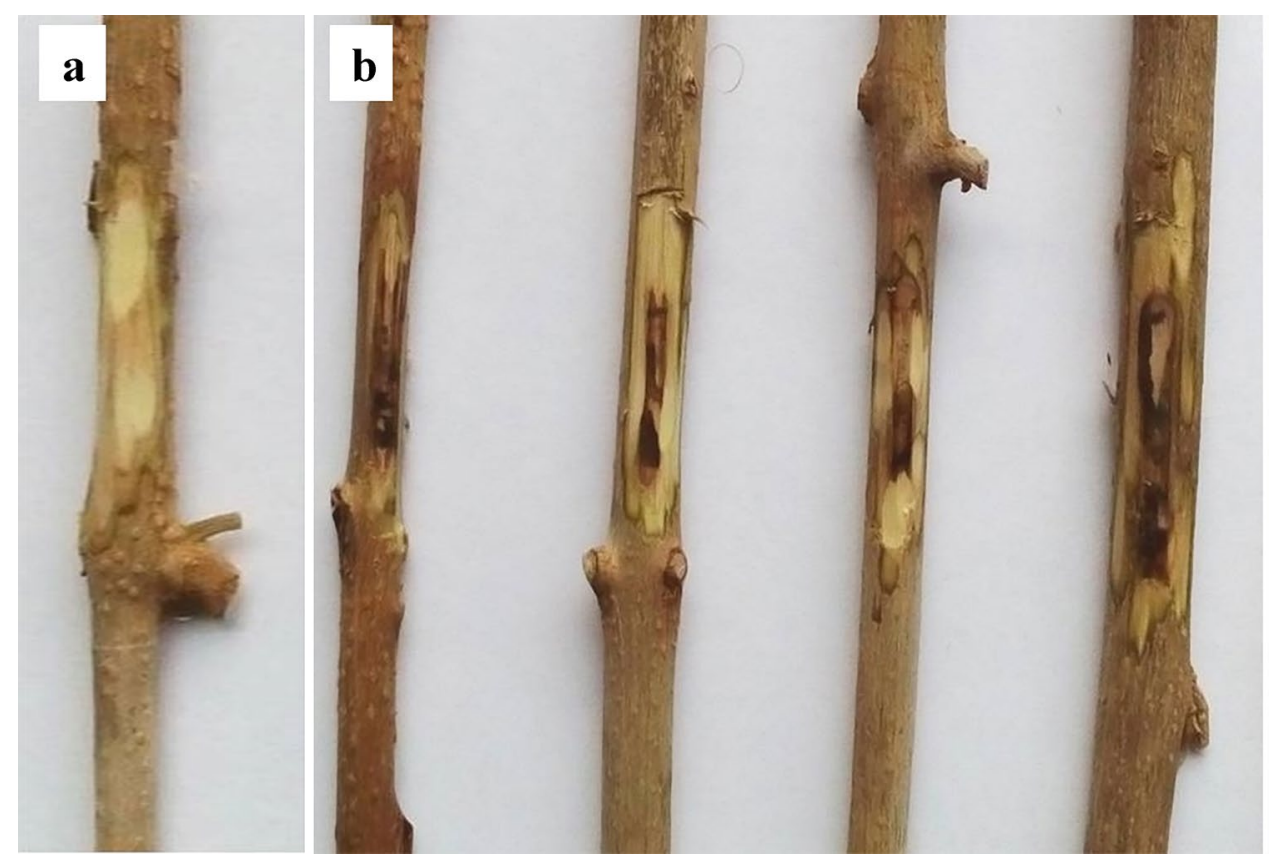


lesion length, depth and colour. The lesions caused by the three $A$. alternata isolates were significantly different from the control but not from each other. Isolations from the controls were sterile. However, the isolates derived from artificially inoculated cuttings were amplified and the amplicons were sequenced. A BLAST search of the Genbank database revealed $100 \%$ homology with various A. alternata isolates. The above described procedure fulfilled Koch's postulates.

Alternaria alternata has been reported previously causing leaf spots on olive trees (Bourbos et al. 1999) and bud and blossom blight of olive trees (Lagogianni et al. 2017) in Greece. The same fungus has been also isolated from wilted olive trees in Italy and from olive fruits and leaves in Pakistan (Farr and Rossman 2019). To our knowledge, this is the first report of this pathogen as causal agent of necrosis on $O$. europaea cuttings in Greece. The significance of the present report is in the difference from the previous reported diseases caused by A. alternata on olive and on its potential to necrotize a great number of cuttings grown in nurseries, the main material for olive propagation in the country.

Acknowledgements The authors gratefully acknowledge Dr. Irene Vloutoglou and Mr. Dimitrios Tsirogiannis, Benaki Phytopathological Institute (Athens, Greece) for providing the Collection Accession No.

\section{References}

Andrew M, Peever TL, Pryor BM (2009) An expanded multilocus phylogeny does not resolve morphological species within the small-spored Alternaria species complex. Mycol 101(1):95-109
Bourbos VA, Skoundridakis MT, Metzidakis I (1999) Alternaria alternata: a new disease of leafy cuttings of olive shoots. Acta Hort 474:585-588

Carbone I, Kohn LM (1999) A method for designing primer sets for speciation studies in filamentous ascomycetes. Mycol 91:553-556

Dhingra OB, Sinclair JB (1995) Basic Plant Pathology Methods. CRC Press, Boca Raton

Farr DF, Rossman AY (2019) Fungal Databases, U.S. National Fungus Collections, ARS, USDA. Retrieved September 13, 2020, from https:// nt.ars-grin.gov/fungaldatabases/

IOC (2012) General Description of Olive Growing in Greece. Available online: https://www.inter nationaloliveoil.org/wpcontent/ uploads/2019/11/ENGLISH_POLIC Y_GREECE-2012_ OK.pdf (Accessed on 13 Sept 2020)

Lagogianni CS, Tjamos EC, Antoniou PP, Tsitsigiannis DI (2017) First report of Alternaria alternata as the causal agent of Alternaria bud and blossom blight of olives. Pl Dis 101(12):2151-2152

Simmons EG (2007) Alternaria: an Identification Manual. APS Press, St. Paul, MN

Torres M, Pierantozzi P, Searles P, Rousseaux C, García-Inza G, Miserere A, Bodoira R, Contreras C, Maestri D (2017) Olive cultivation in the Southern Hemisphere: Flowering, water requirements and oil quality responses to new crop environments. Front Plant Sci 8:1830

White TJ, Bruns T, Lee S, Taylor J (1990) Amplification and direct sequencing of fungal ribosomal RNA genes for phylogenetics. In 'PCR protocols: a guide to methods and applications'. (Eds MA Innis, DH Gelfand, JJ Snindky, TJ White). pp 315-322. Academic Press, San Diego, California

Woudenberg JHC, Seidl MF, Groenewald JZ, de Vries M, Stielow JB, Thomma BPHJ, Crous PW (2015) Alternaria section Alternaria: species, formae speciales or pathotypes? Studies in Mycol $82: 1-21$ 